\title{
A Minimax-Measure Intersection Problem
}

\author{
Philip R. Meyers \\ Institute for Basic Standards, National Bureau of Standards, Washington, D.C. 20234
}

(March 16, 1976)

\begin{abstract}
The problem solved is that of selecting $n$ subsets of the unit interval, each of measure $\alpha$, so as to minimize the maximum of the measures of their $p$-fold intersections. This is achieved by minimizing the sum of the measures of these $p$-fold intersections.
\end{abstract}

Key words: Combinatorial analysis; combinatorial probability; measure theory; minimax.

\section{Introduction}

Some years ago, NBS colleague S. Haber communicated the following problem: To select $n$ subsets of the unit interval, each of measure $1 / 2$, so as to minimize the maximum of the measures of the pairwise intersections of these subsets. The problem is suggested by a paper [1] $]^{1}$ of Gillis which, settling "an unpublished conjecture of Erdos," proves that for denumerably infinite collections of sets of measure $\alpha$, the value corresponding to the maximum pairwise-intersection measure has infimum $\alpha^{2}$. (Collections with higher transfinite cardinality are treated by Gillis in [2].) Here we provide an explicit solution for collections of finite cardinalities $n$. Further, and also corresponding to [1], we consider as well the case of $p$-fold intersections with $2 \leq p \leq n$, and provide the corresponding explicit solution. (As noted in [2], the argument of [1] easily extends to show that $\alpha^{P}$ is the limiting value for a denumerably infinite collection.)

As preliminary, we introduce a second minimization and point out its relationship to our minimax problem, to wit: Select $n$ subsets $A_{1}, A_{2}$, . ., $A_{n}$ of the unit interval, each of measure $\alpha$, so that the sum of the measures of their $p$-fold intersections is minimum. If now $X=\left\{S_{1}, \ldots, S_{n}\right\}$, a solution to this minimum problem, can be chosen so that all its $p$-fold intersections have the same measure $s$, and if $M$ is the maximum of the measures of the $p$-fold intersections of an arbitrary collection $A_{1}, A_{2}$, $\ldots, A_{n}$ with all $\mu\left(A_{i}\right)=\alpha$, then

$$
\begin{aligned}
\left(\begin{array}{c}
n \\
p
\end{array}\right) M & \geq \sum_{i_{1}<i_{2}<\ldots<i_{p}} \mu\left(A_{i_{1}} \cap A_{i_{2}} \cap \ldots \cap \mathrm{A}_{i_{p}}\right) \\
& \geq_{i_{1}<i_{2}<} \ldots<i_{p} \mu\left(S_{i_{1}} \cap S_{i_{2}} \cap \ldots \cap S_{i_{p}}\right) \\
& =\left(\begin{array}{c}
n \\
p
\end{array}\right) s .
\end{aligned}
$$

Thus $\mathrm{s} \geq M$, demonstrating that $X$ solves the minimax problem. This observation suggested the analysis which follows.

AMS Subject Classification: Primary 05A05; Secondary 05B99, 10E30, 28 A75.

${ }^{1}$ Figures in brackets indicate the literature references at the end of this paper. 


\section{Analysis}

We will use the following notation: $N=\{1,2, \ldots, n\} ; p$ is a fixed positive integer with $2 \leq p \leq n$. The underlying space is the unit interval $I$ with Lebesgue measure $\mu$ (but the analysis actually carries over to any "atomless" probability space). Set complements of $A \subseteq I$ and $R \subseteq N$ are denoted $A^{c}$ and $R^{c}$ respectively. Let, for $0 \leq r \leq n$,

$$
K_{r}=\{R \subseteq N: \quad|R|=r\} .
$$

Given the real number $\alpha$ with $0<\alpha \leq 1$, let

$$
F(\alpha)=\{A \subseteq I: \mu(A)=\alpha\}
$$

and let $F^{n}(\alpha)$ denote the $n$-fold Cartesian power of $F(\alpha)$, consisting of all $n$-tuples

$$
X=\left\{A_{1}, A_{2}, \ldots, A_{n}\right\}
$$

with each $A_{i} \in F(\alpha)$. For each such $X$, and each $R \subseteq N$, set

$$
X_{R}=\left\{x \in I: \quad x \in A_{i} \quad \text { iff } i \in R\right\}
$$

an easily-proved property of these sets, to be used repeatedly below, is that

$$
X_{R} \cap\left(\cap_{i \in P} A_{i}\right)=\left\{\begin{array}{l}
X_{R} \text { if } P \subseteq R, \\
\phi \text { otherwise. }
\end{array}\right.
$$

Note that the disjoint union

$$
X_{r}=\underset{R \in K_{r}}{\cup} \mathrm{X}_{R}
$$

consists of those points $x \in I$ which are members of exactly $r$ sets $A_{i} \in X$. Finally, for measurable $B$ 
$\subseteq I$, it is convenient to define

$$
S(X, B)=\sum_{P \in K_{p}} \mu\left[\left(\cap_{i \in P} A_{i}\right) \cap B\right] .
$$

The "objective function" for the minimax problem is

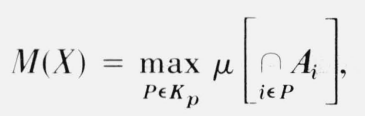

while that for the related minimization problem introduced in section 1 is

$$
S(X)=S(X, I)=\sum_{P \in K_{p}} \mu\left[\cap_{i \in P} A_{i}\right] .
$$

An alternative formula for $S(X)$ will first be developed (Lemma 1), and then a necessary condition (Lemma 2) for some $X \in F^{n}(\alpha)$ to minimize $S$ will be presented.

Lemma 1: For each $\mathrm{X} \in \mathrm{F}^{n}(\alpha)$,

$$
\mathrm{S}(\mathrm{X})=\sum_{r=p}^{n}\left(\begin{array}{l}
\mathrm{r} \\
\mathrm{p}
\end{array}\right) \mu\left(\mathrm{X}_{\mathrm{r}}\right) .
$$

Proof: Since $\left\{X_{r}: \quad r=0,1, \ldots, n\right\}$ is a partition of $I$,

$$
\begin{aligned}
S(X) & =\sum_{r=0}^{n} S\left(X, X_{r}\right) \\
& =\sum_{r=0}^{n} \sum_{|R|=r} S\left(X, X_{R}\right) .
\end{aligned}
$$

Applying (1) to each summand, we obtain

$$
S(X)=\sum_{r=p}^{n} \sum_{|R|=r}\left(\begin{array}{l}
r \\
p
\end{array}\right) \mu\left(X_{R}\right),
$$

yielding (2). 
Lemma 2. If $\mathrm{X}$ minimizes $\mathrm{S}$ over $\mathrm{F}^{\mathrm{n}}(\alpha)$, and $\mu\left(\mathrm{X}_{\mathrm{r}}\right)>0$ for some $\mathrm{r} \geq \mathrm{p}$, then $\mu\left(\mathrm{X}_{\mathrm{t}}\right)=0$ for all $\mathrm{t}$ $<\mathrm{r}-1$.

Proof: Suppose, to the contrary, that there exist $r \geq p$ and $t<r-1$ such that $\mu\left(X_{r}\right)>0$ and $\mu\left(X_{t}\right)>0$. We will prove the existence of an $X^{\prime} \in F^{n}(\alpha)$ for which $S\left(X^{\prime}\right)<S(X)$, thus contradicting the hypothesis about $X$.

Since $\mu\left(X_{r}\right)>0$ and $\mu\left(X_{t}\right)>0, K_{r}$ and $K_{t}$ must contain respective members $R$ and $T$ with $\mu\left(X_{R}\right)$ $>0$ and $\mu\left(X_{T}\right)>0$. Choose subsets $Y$ and $Z$ of $I$ with

$$
Y \subseteq X_{R}, \quad Z \subseteq X_{T}, \quad \mu(Y)=\mu(Z)>0 .
$$

Also choose a member $i$ of the nonempty set $R-T$; then

$$
Y \subseteq A_{i} ; \quad Z \subseteq A_{i}^{c}
$$

Now define $X^{\prime}=\left\{A_{1}, A_{2}, \ldots, A_{i}^{\prime}, \ldots, A_{n}\right\}$, where

$$
A_{i}^{\prime}=\left(\mathrm{A}_{i}-\mathrm{Y}\right) \cup \mathrm{Z}
$$

Since $\mu\left(A_{i}^{\prime}\right)=\mu\left(\mathrm{A}_{i}-Y\right)+\mu(Z)=\mu\left(A_{i}\right)$, we have $X^{\prime} \epsilon F^{\prime \prime}(\alpha)$.

To prove that $\left.S(X)^{\prime}\right)<S(X)$, observe that $I$ is partitioned into $Y, Z$, and $I-Y-Z$.

Thus

$$
\begin{gathered}
S(X)=S(X, Y)+S(X, Z)+S(X, I-Y-Z), \\
S\left(X^{\prime}\right)=S\left(X^{\prime}, Y\right)+S\left(X^{\prime}, Z\right)+S\left(X^{\prime}, I-Y-Z\right) .
\end{gathered}
$$

Since $X$ and $X^{\prime}$ differ only on $Y \cup Z$, it follows that

$$
S(X)-S\left(X^{\prime}\right)=\left[S(X, Y)-S\left(X^{\prime}, Y\right)\right]-\left[S\left(X^{\prime}, Z\right)-S(X, Z)\right] .
$$

Since $Y \subseteq X_{r}$ and $Y \subseteq X^{\prime}{ }_{r-1}$, application of (1) to the summands of $S(X, Y)$ and $S\left(X^{\prime}, Y\right)$ yields

$$
S(X, Y)-S\left(X^{\prime}, Y\right)=\left(\begin{array}{c}
r \\
p
\end{array}\right) \mu(Y)-\left(\begin{array}{c}
r-1 \\
p
\end{array}\right) \mu(Y)=\left(\begin{array}{c}
r-1 \\
p-1
\end{array}\right) \mu(Y) .
$$


Similarly, it follows from $Z \subseteq X_{t}$ and $Z \subseteq X^{\prime}{ }_{t+1}$ that

$$
S\left(X^{\prime}, Z\right)-S(X, Z)=\left(\begin{array}{c}
t+1 \\
p
\end{array}\right) \mu(Z)-\left(\begin{array}{c}
t \\
p
\end{array}\right) \mu(Z)=\left(\begin{array}{c}
t \\
p-1
\end{array}\right) \mu(Z)
$$

Since $r-1>t$ and $\mu(Y)=\mu(Z)>0$,

$$
S(X)-S\left(X^{\prime}\right)=\left(\begin{array}{l}
r-1 \\
p-1
\end{array}\right) \mu(Y)-\left(\begin{array}{c}
t \\
p-1
\end{array}\right) \mu(Z)>0
$$

completing the proof.

We will subsequently show that if $\mu\left(X_{r}\right)>0$ for some $r>p$ then $\mu\left(X_{t}\right)=0$ for $t<r-1$ is a sufficient condition for $X$ to minimize $S$ over $F^{n}(\alpha)$.

Lemma 3: For all $\mathrm{X} \in \mathrm{F}^{\mathrm{n}}(\alpha)$,

$$
\mathrm{n} \alpha=\sum_{\mathrm{r}=0}^{\mathrm{n}} \mu\left(X_{r}\right)
$$

Proof: Let $c_{i}$ denote the characteristic function of $A_{i}$. Then

$$
\begin{aligned}
n \alpha & =\sum \sum_{i=1}^{n} \int_{I} c_{i}(x) d \mu(x)=\int_{I}\left[\sum_{i=1}^{n} c_{i}(x)\right] d \mu(x) \\
& =\sum_{r=0}^{n} \int_{X_{r}}\left[\sum_{i=1}^{n} c_{i}(x)\right] d \mu(x)=\sum_{r=0}^{n} r \mu\left(X_{r}\right) .
\end{aligned}
$$

It is now possible to prove:

Lemma 4: If $n \alpha \leq p-1$, then $S_{\min }=\min \left\{S(Y): \quad Y \in F^{n}(\alpha)\right\}=0$.

Proof: It suffices to exhibit an $X \in F^{n}(\alpha)$ for which

To this end, let

$$
\mu\left(X_{r}\right)=0 \quad \text { for } r \geq p
$$

$$
A_{i}=[(i-1) \alpha, i \alpha) \quad(\bmod 1) \quad \text { for } 1 \leq i \leq n .
$$

Each point of $[0,1)$ corresponds $(\bmod 1)$ to exactly $p-1$ points of the interval $[0, p-1)$, and thus to at most $p-1$ points of the subinterval $[0, n \alpha)$; thus $X_{r} \cap[0,1)=\phi$ for $r \geq p$, verifying (4).

LEMMA 5: If for given $\mathrm{X} \in \mathrm{F}^{\mathrm{n}}(\alpha)$, the largest $\mathrm{r}$ such that $\mu\left(\mathrm{X}_{\mathrm{r}}\right)>0$ satisfies $\mathrm{r} \geq \mathrm{p}$ and further for $\mathrm{t}<\mathrm{r}-1, \mu\left(\mathrm{x}_{\mathrm{t}}\right)=0$, then

$$
\mathrm{S}(\mathrm{X})=\mathrm{S}_{\min }
$$

Proof. It suffices to show that $S(X)$ has the same value for all $X \in F^{n}(\alpha)$ satisfying the conditions of the lemma. Consider such an $X$, and the greatest $r$ for which $\mu\left(X_{r}\right)>0$. Since

$$
\sum_{t=0}^{n} \mu\left(X_{t}\right)=\mu(I)=1
$$

such an $r$ must exist. By the above condition $\mu\left(X_{t}\right)=0$ for $t \neq r, r-1$, and so by (5), 


$$
\mu\left(X_{r-1}\right)=1-\mu\left(X_{r}\right)
$$

Let $n \alpha=m+\beta$ with $m$ integral and $0 \leq \beta<1$. It follows from (3) that

$$
m+\beta=r \mu\left(X_{r}\right)+(r-1) \mu\left(X_{r-1}\right)=(r-1)+\mu\left(X_{r}\right),
$$

and from (2) that

$$
S(X)=\left(\begin{array}{c}
r \\
p
\end{array}\right) \mu\left(X_{r}\right)+\left(\begin{array}{c}
r-1 \\
p
\end{array}\right) \mu\left(X_{r-1}\right)=\left(\begin{array}{c}
r-1 \\
p
\end{array}\right)+\left(\begin{array}{c}
r-1 \\
p-1
\end{array}\right) \mu\left(X_{r}\right) .
$$

If $\beta=0$, then since $m$ is integral and $0<\mu\left(X_{r}\right) \leq 1$, it follows from (6) that $\mu\left(X_{r}\right)=1$ and $r=$ $m$, and then it follows from (7) that

$$
S(X)=\left(\begin{array}{c}
m-1 \\
p
\end{array}\right)+\left(\begin{array}{c}
m-1 \\
p-1
\end{array}\right)=\left(\begin{array}{l}
m \\
p
\end{array}\right) .
$$

If $\beta>0$, then it follows from (6) that $\mu\left(X_{r}\right)=\beta$ and $m=r-1$, and then it follows from (7) that

$$
S(X)=\left(\begin{array}{c}
m \\
p
\end{array}\right)+\left(\begin{array}{c}
m \\
p-1
\end{array}\right) \beta .
$$

Thus $S(X)$ is uniquely determined by the pair $(m, \beta)$, i.e., by $n \alpha$. Note that (8) and (9) are consistent with Lemma 4 , since both yield $S(X)=0$ if $n \alpha \leq p-1$.

We are now able to provide the solutions, both to the problem of minimizing $S(X)$ over $F^{n}(\alpha)$ and to the original problem of minimizing

$$
M(X)=\max _{P \in K_{p}} \mu\left(\cap_{i \in P} A_{i}\right)
$$

over $F^{n}(\alpha)$. Let $M_{\min }$ denote the value of this latter minimum. Then the solution takes the following form.

Theorem. Let $\mathrm{n} \alpha=\mathrm{m}+\beta$ with $\mathrm{m}$ integral and $0 \leq \beta<1$. Then

$$
\begin{array}{ll}
\mathrm{S}_{\min }=\mathrm{M}_{\min }=0 & \text { if } \mathrm{n} \alpha \leq \mathrm{p}-1, \\
\mathrm{~S}_{\min }=\left(\begin{array}{c}
\mathrm{m} \\
\mathrm{p}
\end{array}\right)+\left(\begin{array}{c}
\mathrm{m} \\
\mathrm{p}-\mathrm{l}
\end{array}\right) \beta, \quad \mathrm{M}_{\min }=\mathrm{S}_{\min } /\left(\begin{array}{l}
\mathrm{n} \\
\mathrm{p}
\end{array}\right) & \text { if } \mathrm{n} \alpha>\mathrm{p}-1 .
\end{array}
$$

Thus, in particular, for the problem as originally posed where $\mathrm{p}=2$ and $\alpha=1 / 2$,

$$
\mathrm{S}_{\min }=\left(\begin{array}{ll}
\mathrm{k}(\mathrm{k}-1) / 2 & \text { if } \mathrm{n}=2 \mathrm{k} \\
(\mathrm{k}-1)^{2} / 2 & \text { if } \mathrm{n}=2 \mathrm{k}-1
\end{array}\right.
$$

and $\mathrm{M}_{\min }=(\mathrm{k}-1) / 2(2 \mathrm{k}-1)$.

Proof: First suppose $n \alpha \leq p-1$. Then $S_{\min }=0$ follows from Lemma 4 , whose proof constructed an $X \in F^{n}(\alpha)$ for which $\mu\left(\cup_{r=p}^{n} X_{r}\right)=0$. Since every $p$-fold intersection of the members of $X$ lies in this union, it follows that $M(X)=0$, implying $M_{\min }=0$. 
Now suppose $n \alpha>p-1$. The formula for $S_{\min }$ follows from (8) and (9). We will prove the result for $M_{\min }$ by constructing an $X \in F^{n}(\alpha)$ which satisfies the condition of Lemma 2, and which furthermore (see the end of sec. 1) has equal measures for each of its $p$-fold intersections. For this purpose, partition the interval $[0, \beta)$ into $\left(\begin{array}{c}n \\ m+1\end{array}\right)$ equal subintervals and the interval $[\beta, 1]$ into $\left(\begin{array}{c}n \\ m\end{array}\right)$ equal subintervals. Label the second family of subintervals as $\left\{X_{M}: M \in K_{m}\right\}$ and the first family as $\left\{X_{Q}: \quad Q \in K_{m+1}\right\}$. Define

$$
A_{i}=\left[\cup\left\{X_{M}: \quad i \in M\right\}\right] \cup\left[\cup\left\{X_{Q}: \quad i \in Q\right\}\right] .
$$

Then each $A_{\mathrm{i}}$ consists of $\left(\begin{array}{c}n-1 \\ m-1\end{array}\right)$ intervals $X_{M}$ and $\left(\begin{array}{c}n-1 \\ m\end{array}\right)$ intervals $X_{Q}$, all disjoint, so all $A_{i}$ have equal measure. If $c_{i}$ denotes the characteristic function of $A_{i}$, then

$$
\begin{aligned}
\sum_{i=1}^{n} \mu\left(A_{i}\right) & =\int_{I}\left(\sum_{i=1}^{n} c_{i}\right) d \mu=\int_{0}^{\beta}\left(\sum_{i=1}^{n} c_{\mathrm{i}}\right) d \mu+\int_{\beta}^{1}\left(\sum_{i=1}^{n} c_{i}\right) d \mu \\
& =(m+1) \beta+m(1-\beta)=m+\beta=\mathrm{n} \alpha .
\end{aligned}
$$

Thus each $\mu\left(A_{\mathrm{i}}\right)=\alpha$, i.e., $X \in F^{n}(\alpha)$. For $r \geq p, \mu\left(X_{r}\right)>0$ holds only for $r=m$ and $r=m+1$, so the condition of Lemma 2 is satisfied. The symmetry of the construction assures that all $p$-fold intersections of the members of $X$ have equal measure; explicitly, for $P \in K_{p}$, we have

$$
\cap_{i \in P} A_{i}=\left[\cup\left\{X_{M}: P \subset M\right\}\right] \cup\left[\cup\left\{X_{Q}: P \subset Q\right\}\right] .
$$

implying

$$
\left.\underset{i \in P}{\mu} A_{i}\right]=\left(\begin{array}{c}
n-p \\
m-p
\end{array}\right)(1-\beta) /\left(\begin{array}{c}
n \\
m
\end{array}\right)+\left(\begin{array}{c}
n-p \\
m+1-p
\end{array}\right) \beta /\left(\begin{array}{c}
n \\
m+1
\end{array}\right),
$$

independently of $P$.

\section{References}

[1] Gillis, J., Note on a property of measurable sets, J. London Math. Soc. 11, 139-141 (1936).

[2] Gillis, J., Some combinatorial properties of measurable sets, Quart. J. Math. (Oxford) 7, 191-198 (1936).

(Paper 80B2-438) 